\title{
Dieta de Leptodactylus latrans (Steffen, 1815) na Serra do Sudeste, Rio Grande do Sul, Brasil
}

\author{
Daiane Maria Melo Pazinato ${ }^{1}$ \\ Adriane de Oliveira Trindade ${ }^{2}$ \\ Stefan Vilges de Oliveira ${ }^{3 *}$ \\ Lize Helena Cappellari ${ }^{1}$ \\ ${ }^{1}$ Universidade da Região da Campanha - URCAMP, Campus Caçapava do Sul \\ CEP 96570-000, Caçapava do Sul - RS, Brasil \\ ${ }^{2}$ Universidade Federal do Pampa - UNIPAMPA, Caçapava do Sul - RS, Brasil \\ ${ }^{3}$ Organização Não Governamental Interação de trabalhos Ambientais - ITA \\ Caixa Postal 05, Caçapava do Sul - RS, Brasil \\ *Autor para correspondência \\ stefanbio@yahoo.com.br
}

Submetido em 19/02/2011

Aceito para publicação em 05/08/2011

\section{Resumo}

É descrita quantitativamente e qualitativamente a dieta de Leptodactylus latrans baseado no conteúdo estomacal de 37 espécimes procedentes do município de Caçapava do Sul, RS. Os indivíduos analisados foram coletados entre janeiro de 2007 e fevereiro de 2008. A dieta é composta por 17 categorias de presas. Coleoptera e Araneae foram os itens mais frequentes com 55\% e 22,5\%. Volumetricamente, os itens mais importantes foram Crustacea, Decapoda (15,68\%) e Amphibia (13,75\%).

Palavras-chave: Amphibia, Dieta, Leptodactylus latrans, Rio Grande do Sul

\section{Abstract}

Diet of Leptodactylus latrans from Serra do Sudeste, Rio Grande do Sul, Brazil. The diet of Leptodactylus latrans is described based on the stomach contents of 37 specimens from the Caçapava do Sul municipality, in the state of Rio Grande do Sul, Brazil. The individuals were sampled between January 2007 and February 2008. The diet was composed of 17 types of prey, and Coleoptera (55\%) and Araneae $(22.5 \%)$ were the most frequent items. Volumetrically, the most important items were Crustacea, Decapoda (15.68\%), and Amphibia (13.75\%).

Key words: Amphibia, Diet, Leptodactylus latrans, Rio Grande do Sul 


\section{Introdução}

Em território brasileiro são encontradas 849 espécies de anuros (SBH, 2011), o que torna o país o mais diverso do mundo para o grupo. Entretanto, o conhecimento sobre a biologia e ecologia da maioria das espécies é escasso, principalmente nas regiões interioranas, pois, historicamente, os estudos têm se desenvolvido principalmente na zona litorânea e ao longo dos grandes rios (HADDAD et al., 1988). Hensel (1867) foi um dos primeiros a estudar os anfíbios da região Sul do Brasil, elaborando uma lista de 22 espécies para o Estado do Rio Grande do Sul. Braun e Braun (1980) apresentaram uma lista com 65 espécies e, recentemente, Borges-Martins et al. (2007) informam a ocorrência de aproximadamente 93 espécies, o que corresponde a $11 \%$ do total de anuros que ocorrem no Brasil.

O gênero Leptodactylus apresenta 89 espécies distribuídas principalmente na América do Sul (FROST, 2011). Em recente estudo, Lavilla et al. (2010) revalidaram a espécie Leptodactylus latrans (Steffen, 1815), anteriormente designada como Leptodactylus ocellatus. Esta espécie é a maior rã do Rio Grande do Sul, podendo atingir $15 \mathrm{~cm}$ de comprimento rostro-cloacal e pesar até $350 \mathrm{~g}$. Geralmente habitam corpos de água lênticos e córregos em áreas abertas (FREITAS; SILVA, 2004; BORGES-MARTINS et al., 2007).

O presente trabalho apresenta a dieta de uma população de Leptodactylus latrans de uma área na Serra do Sudeste, Município de Caçapava do Sul, Rio Grande do Sul, Brasil.

\section{Material e Métodos}

O trabalho foi realizado no Município de Caçapava do Sul, Rio Grande do Sul (53²9'16”W, 30³1'11'S). A área de estudo faz parte do Bioma Pampa, região zoogeográfica da província Pampeana da Serra do Sudeste. Esta região é uma fisionomia de transição, por apresentar um mosaico de formações herbáceoarbustivas e florestais (CORDEIRO; HASENACK, 2009). O clima no Município de Caçapava do Sul é classificado como temperado úmido, com precipitação pluvial média de $1588 \mathrm{~mm}$. A temperatura média do mês mais frio é de $11^{\circ} \mathrm{C}$ e a média anual é de $16,8^{\circ} \mathrm{C}$ (MALUF, 2000).

Foram realizadas amostragens através da busca por procura visual, procurando sistematicamente pelos indivíduos. Os espécimes foram capturados manualmente em ambientes lênticos (banhados e açudes), entre janeiro de 2007 e fevereiro de 2008.

Foram coletados 37 indivíduos que foram mortos no momento da captura, através de injeção letal do anestésico Tiopental ${ }^{\circledR}$, fixados com formol a $10 \%$ e conservados em álcool 70\%. Posteriormente foram medidos $(\mathrm{CRC})$ com paquímetro Mitutoyo $\odot$ (precisão de $0,01 \mathrm{~mm}$ ) e dissecados. Seus conteúdos estomacais foram analisados sob estereomicroscópio. Os espécimes foram depositados na coleção do Museu de Ciências e Tecnologia da Pontifícia Universidade Católica do Rio Grande do Sul (MCP 9825 - MCP 9861).

A abundância e a frequência absolutas das categorias alimentares foram obtidas através da contagem do número de itens presentes nos estômagos. O comprimento e a largura dos itens foram medidos para estimar seu volume, segundo a fórmula dos volumes esferóides (CALDWELL; VITT, 1999):

$$
\mathrm{V}=\frac{4}{3} \cdot \pi \cdot\left(\frac{\text { compriment } \mathrm{o}}{2}\right) \cdot\left(\frac{\text { largura }}{2}\right)^{2}
$$

A diversidade de presas encontrada nos estômagos foi calculada pelo índice de Shannon-Wiener (PIANKA, 1973): $\mathrm{H}^{\prime}=-\Sigma p_{i} \log _{2}\left(p_{i}\right)$, sendo $p_{i}$ a abundância relativa do táxon de presa $i$ na dieta.

Para determinar o grau de semelhança entre dietas de fêmeas e machos foi utilizado o índice de sobreposição de nicho (PIANKA, 1973):

$$
\begin{aligned}
& \mathrm{O}_{\mathrm{jk}} \sum P_{i j} P_{i k} \\
& \sqrt{\sum \mathrm{P}_{\mathrm{ij}}{ }^{2} \sum \mathrm{P}_{i k}{ }^{2}}
\end{aligned}
$$

Onde $\mathrm{P}_{\mathrm{ij}}$ e $\mathrm{P}_{\mathrm{ik}}$ são as proporções dos itens da categoria i nas espécies j e k. 
Para descrever a importância de cada item consumido, foi calculado o Índice de Importância Relativa (PINKAS et al., 1971): IRIt $=(\mathrm{POt}) \times(\mathrm{PIt}+$ $\mathrm{PVt}$ ), onde POt é a porcentagem de ocorrência (100 x número de estômagos contendo o item $\mathrm{t}$ /número total de estômagos), PIt é a porcentagem de indivíduos (100 $\mathrm{x}$ número de indivíduos $\mathrm{t}$ em todos estômagos/número de indivíduos de todos os taxa em todos os estômagos), e PVt é a porcentagem do volume (100 x volume total de indivíduos $\mathrm{t}$ em todos estômagos/volume total em todos estômagos).

\section{Resultados e Discussão}

Dos 37 estômagos de Leptodactylus latrans analisados, 22 eram de machos e 15 eram de fêmeas. A dieta é composta por 17 itens. Coleoptera foi o item mais frequente, ocorrendo em 55\% dos estômagos, correspondendo a $31,96 \%$ do número total de presas ingeridas $(n=97)$ e $9,86 \%$ do volume total de presas. $\mathrm{O}$ segundo item mais frequente foi Araneae (22,5\%), correspondendo a $8,93 \%$ do número total de presas e com $1,58 \%$ do volume total. Volumetricamente, os itens mais importantes foram Decapoda (15,68\% do volume total), seguido de Amphibia (13,75\%) (Tabela 1).

A diversidade média de presas encontradas por estômago foi de 2,18 $\pm 1,10$ e a riqueza máxima encontrada em um estômago foi de cinco categorias diferentes. O número máximo de itens encontrados em um único estômago foi sete, e a média geral foi de 2,85 $\pm 1,54$ itens. O número médio de presas consumidas por machos foi $2,35 \pm 1,27$, e de fêmeas foi $3,57 \pm 1,65$. O volume médio de presas consumidas por machos foi de $3.066,47 \pm 3.348,24 \mathrm{~mm}^{3}$, por fềmeas foi de $4.695,30 \pm$ $5.123,43 \mathrm{~mm}^{3}$ (Tabela 1 ).

Em machos o item mais representativo volumetricamente foi Orthoptera, com 30,04\% do volume total, enquanto em fêmeas foi Decapoda, com $37,83 \%$ do volume total. Tanto em machos como em fêmeas, exemplares de Coleoptera foram as presas mais comuns, correspondendo a respectivamente, 34\% e $30 \%$ do total, sendo também o item mais frequente em ambos os sexos (30,55\% em machos e $24,32 \%$ em fêmeas) (Tabela 1).
A similaridade alimentar quanto ao número de presas entre machos e fêmeas foi elevada $\left(\mathrm{O}_{\mathrm{j} k}=0,93\right)$. No entanto, foi baixa considerando o volume de presas $\left(\mathrm{O}_{\mathrm{jk}}\right.$ $=0,40)$. $\mathrm{O}$ índice de importância relativa mostrou que Coleoptera foi o item mais importante na dieta de fêmeas $(I R I=1,01)$ e de machos $(I R I=1,43)$. A diversidade de presas consumidas foi maior nas fêmeas $\left(\mathrm{H}^{\prime}=3,31\right)$ do que nos machos $\left(\mathrm{H}^{\prime}=3,18\right)$.

O item mais numeroso da dieta de Leptodactylus latrans foi Coleoptera, correspondendo a $30 \%$ das presas consumidas. Estes valores são similares aos encontrados por Teixeira e Vrcibradic (2003), França et al. (2004) e Maneyro et al. (2004). Entretanto, volumetricamente, os itens mais importantes foram Decapoda $(15,68 \%)$ e Anura (13,75\%). A despeito da pouca representatividade numérica destes itens, seu grande tamanho em relação aos demais itens alimentares explica sua relevância na dieta.

Apesar de Decapoda ter constituído um item volumetricamente representativo na dieta, parece ser consumida oportunisticamente. O único registro de seu consumo por Leptodactylus latrans foi apresentado por Solé et al. (2009), que citam a ocorrência deste item em apenas um exemplar de L. latrans, amostrado em uma plantação de cacau na Bahia.

O índice de importância relativa (IRI) mostra que os maiores valores encontrados corresponderam a Coleoptera $(1,43)$ e a Scorpiones $(0,66)$ em machos e a Coleoptera $(1,01)$ e Amphibia $(0,61)$, em fêmeas. A presença de escorpiões na dieta de $L$. latrans parece não ser comum, pois não foram encontrados registros de seu consumo na literatura.

Machos e fêmeas apresentaram uma similaridade trófica maior numericamente do que volumetricamente, provavelmente devido ao fato das fêmeas terem se alimentado de um número maior de Decapoda e de Anuros, em relação aos machos. O grande volume destes itens pode explicar essa diferença na dieta entre machos e fêmeas.

Em apenas 5\% dos estômagos analisados foram encontrados Anuros, o que significa pouca representatividade na dieta de L. latrans. Este item, entretanto, foi o segundo de maior importância relativa na dieta das fêmeas, devido ao seu grande volume. 
TABELA 1: Dieta de Leptodactylus latrans. N: número total de presas, V: volume, F: frequência, IRI: índice de relativa importância.

\begin{tabular}{|c|c|c|c|c|c|c|c|c|}
\hline \multirow{2}{*}{ Item } & \multicolumn{4}{|c|}{$\operatorname{Machos}(\mathrm{N}=22)$} & \multicolumn{4}{|c|}{ Fêmeas $(\mathrm{N}=15)$} \\
\hline & $\mathrm{N}(\%)$ & $\mathrm{V}(\%)$ & $\mathrm{F}(\%)$ & IRI & $\mathrm{N}(\%)$ & $\mathrm{V}(\%)$ & $\mathrm{F}(\%)$ & IRI \\
\hline \multicolumn{9}{|l|}{ INSECTA } \\
\hline Coleoptera & $\begin{array}{c}16 \\
(34)\end{array}$ & $\begin{array}{c}9.943,09 \\
(24,7)\end{array}$ & $\begin{array}{c}11 \\
(30,5)\end{array}$ & 1,43 & $\begin{array}{c}15 \\
(30)\end{array}$ & $\begin{array}{c}3.522,17 \\
(6,6)\end{array}$ & $\begin{array}{c}9 \\
(24,3)\end{array}$ & 1,01 \\
\hline Hemiptera & $\begin{array}{c}2 \\
(4,3)\end{array}$ & $\begin{array}{c}982,98 \\
(2,4)\end{array}$ & $\begin{array}{c}2 \\
(5,6)\end{array}$ & 0,22 & & & & \\
\hline Hymenoptera & $\begin{array}{c}2 \\
(4,3)\end{array}$ & $\begin{array}{c}1.097,41 \\
(2,7)\end{array}$ & $\begin{array}{c}2 \\
(5,6)\end{array}$ & 0,22 & $\begin{array}{c}1 \\
(2)\end{array}$ & $\begin{array}{c}433,54 \\
(0,8)\end{array}$ & $\begin{array}{c}1 \\
(2,7)\end{array}$ & 0,10 \\
\hline Formicidae & $\begin{array}{c}3 \\
(6,4)\end{array}$ & $\begin{array}{c}72,23 \\
(0,2)\end{array}$ & $\begin{array}{c}2 \\
(5,6)\end{array}$ & 0,22 & $\begin{array}{c}2 \\
(4)\end{array}$ & $\begin{array}{c}166,55 \\
(0,3)\end{array}$ & $\begin{array}{c}2 \\
(5,4)\end{array}$ & 0,19 \\
\hline Orthoptera & $\begin{array}{c}3 \\
(6,4)\end{array}$ & $\begin{array}{c}12.137,77 \\
(30)\end{array}$ & $\begin{array}{c}3 \\
(8,2)\end{array}$ & 0,60 & $\begin{array}{c}4 \\
(8)\end{array}$ & $\begin{array}{c}3.226,13 \\
(6)\end{array}$ & $\begin{array}{c}2 \\
(5,4)\end{array}$ & 0,28 \\
\hline Larvas & $\begin{array}{c}2 \\
(4,3)\end{array}$ & $\begin{array}{c}100,99 \\
(0,2)\end{array}$ & $\begin{array}{c}2 \\
(5,6)\end{array}$ & 0,20 & $\begin{array}{c}2 \\
(4)\end{array}$ & $\begin{array}{c}1.079,99 \\
\text { (2) }\end{array}$ & $\begin{array}{c}2 \\
(5,4)\end{array}$ & 0,20 \\
\hline \multicolumn{9}{|l|}{ CRUSTACEA } \\
\hline Isopoda & $\begin{array}{c}4 \\
(8,5)\end{array}$ & $\begin{array}{c}330,92 \\
(0,8)\end{array}$ & $\begin{array}{c}2 \\
(5,6)\end{array}$ & 0,25 & $\begin{array}{c}2 \\
(4)\end{array}$ & $\begin{array}{c}89,49 \\
(0,2)\end{array}$ & $\begin{array}{c}2 \\
(5,4)\end{array}$ & 0,18 \\
\hline Decapoda & $\begin{array}{c}1 \\
(2,1)\end{array}$ & $\begin{array}{c}1.677,8 \\
(4,1)\end{array}$ & $\begin{array}{c}1 \\
(2,8)\end{array}$ & 0,14 & $\begin{array}{c}3 \\
(6)\end{array}$ & $\begin{array}{c}20.164,83 \\
(37,8)\end{array}$ & $\begin{array}{c}1 \\
(2,7)\end{array}$ & 0,51 \\
\hline \multicolumn{9}{|l|}{ ARACHINIDA } \\
\hline Araneae & $\begin{array}{c}3 \\
(6,4)\end{array}$ & $\begin{array}{c}212,04 \\
(0,5)\end{array}$ & $\begin{array}{c}3 \\
(8,3)\end{array}$ & 0,30 & $\begin{array}{c}6 \\
(12)\end{array}$ & $\begin{array}{c}1.986,60 \\
(3,8)\end{array}$ & $\begin{array}{c}6 \\
(16,2)\end{array}$ & 0,59 \\
\hline Scorpiones & $\begin{array}{c}7 \\
(14,9)\end{array}$ & $\begin{array}{c}8.269,75 \\
(20,6)\end{array}$ & $\begin{array}{c}4 \\
(11)\end{array}$ & 0,66 & $\begin{array}{c}4 \\
(8)\end{array}$ & $\begin{array}{c}2.772,84 \\
(5,2)\end{array}$ & $\begin{array}{c}4 \\
(10,9)\end{array}$ & 0,42 \\
\hline Acari & & & & & $\begin{array}{c}1 \\
(2)\end{array}$ & & $\begin{array}{c}1 \\
(2,7)\end{array}$ & 0,09 \\
\hline Opiliones & $\begin{array}{c}1 \\
(2,1)\end{array}$ & $\begin{array}{c}425,29 \\
(1)\end{array}$ & $\begin{array}{c}1 \\
(2,8)\end{array}$ & 0,11 & & & & \\
\hline CHILOPODA & & & & & $\begin{array}{c}1 \\
(2)\end{array}$ & $\begin{array}{c}84,82 \\
(0,1)\end{array}$ & $\begin{array}{c}1 \\
(2,7)\end{array}$ & 0,09 \\
\hline ANNELIDA & & & & & & & & \\
\hline Oligochaeta & $\begin{array}{c}1 \\
(2,1)\end{array}$ & $\begin{array}{c}1.592,79 \\
(3,9)\end{array}$ & $\begin{array}{c}1 \\
(2,8)\end{array}$ & 0,14 & $\begin{array}{c}2 \\
(4)\end{array}$ & $\begin{array}{c}2.789,96 \\
(5,2)\end{array}$ & $\begin{array}{c}2 \\
(5,4)\end{array}$ & 0,24 \\
\hline MOLLUSCA & & & & & & & & \\
\hline Gastropoda & & & & & $\begin{array}{c}2 \\
(4)\end{array}$ & $\begin{array}{c}1.352,45 \\
(2,6)\end{array}$ & $\begin{array}{c}1 \\
(2,7)\end{array}$ & 0,14 \\
\hline $\begin{array}{l}\text { AMPHIBIA } \\
\text { Anura }\end{array}$ & $\begin{array}{c}2 \\
(4,3) \\
\end{array}$ & $\begin{array}{c}3.539,52 \\
(8,9)\end{array}$ & $\begin{array}{c}2 \\
(5,6) \\
\end{array}$ & 0,28 & $\begin{array}{c}5 \\
(10) \\
\end{array}$ & $\begin{array}{c}15.623,64 \\
(29,4)\end{array}$ & $\begin{array}{c}3 \\
(8,1)\end{array}$ & 0,61 \\
\hline TOTAL & 47 & $40.382,58$ & 36 & & 50 & $53.293,01$ & 37 & \\
\hline
\end{tabular}

Os ácaros foram pouco frequentes já que somente um indivíduo foi encontrado no conteúdo estomacal de uma fêmea, sendo provavelmente uma ingestão ocasional ou acidental. Toft (1981) cita que ácaros, como formigas, apresentam baixo custo de busca, pois são abundantes e lentos em relação a outras presas de anfíbios. Em trabalho com Leptodactylus spixi, Rodrigues et al. (2007) citam o encontro de ácaros em $6,48 \%$ dos estômagos analisados, mas relatam que provavelmente estes ácaros foram ingeridos juntamente aos Orthoptera que estavam parasitando.

Nossos dados mostram que Leptodactylus latrans é uma espécie generalista que consome predominantemente artrópodes, o que corrobora com estudos com esta espécie em outras localidades (STRÜSSMANN et al., 1984; MANEYRO et al., 2004; SANABRIA et al., 2005). 


\section{Referências}

BRAUN, P. C.; BRAUN, C. A. S. Lista prévia dos anfíbios do estado do Rio Grande do Sul, Brasil. Iheringia, Porto Alegre, v. 56 , p. 121-146, 1980 .

BORGES-MARTINS, M.; COLOMBO, P.; ZANKE, C.; BECKER, F. G.; MELO, M. T. Q. 2007. Anfíbios. In: BECKER, F. G.; RAMOS, R. A.; MOURA, L. A. (Ed.). Biodiversidade regiões da lagoa do Casamento e dos Butiazais de Tapes. Planície costeira do Rio Grande do Sul. Porto Alegre: Ministério do Meio Ambiente e Fundação Zoobotânica, 2007. p. 277-291.

CALDWELL, J. P.; VITT, L. J. Dietary asymmetry in leaf litter frogs and lizards in a transitional northern Amazonian rain forest. Oikos, Copenhagen, v. 84, n. 3, p. 383-397, 1999.

CORDEIRO, J. L. P.; HASENACK, H. Cobertura vegetal atual do Rio Grande do Sul. In: PILlAR, V. P.; MULLER, S. C.; CASTILHOS, Z. M. S.; JACQUES, A. V. Á. (Eds). Campos Sulinos, conservação e uso sustentável da biodiversidade. Brasilia: MMA, 2009. p. 285-299.

FRANÇA, L. F.; FACURE, K. G.; GIARETTA, A. A. Trophic and spatial niches of two large-sized species of Leptodactylus (Anura) in Southeastern Brazil. Studies on Neotropical Fauna and Environment, Tübingen, v. 39, n. 3, p. 243-248, 2004.

FREITAS, M. A.; SILVA, T. F. S. Anfíbios na Bahia. Um guia de identificação. Camaçari: Politeno, 2004. 56 p.

FROST, D. Amphibian species of the World 5.5, an online reference. Disponível em: <http://research.amnh.org/vz/ herpetology/amphibia>. Acesso em: 27 maio 2011.

HADDAD, C. F. B; ANDRADE, G. V.; CARDOSO, A. J. Anfíbios anuros do Parque Nacional da Serra da Canastra, estado de Minas Gerais. Brasil Florestal, Brasília, v. 64, p. 9-20, 1988.

HENSEL, R. F. Beittage zur Kentniss der wirbelthiere. Berlin: Arch. Naturgesh, 1867. 120 p.

LAVILLA, E. O.; LANGONE, J. A.; CARAMASCHI, U., HEYER, R.; DE SÁ, R. O. The identification of Rana ocellata Linnaeus, 1758. Nomenclatural impact in the species currently known as Leptodactylus ocellatus (Leptodactylidae) and Osteopilus brunneus (Gosse, 1851) (Hylidae). Zootaxa, Auckland, v. 2346, p. 1-16, 2010.

MALUF, J. R. T. Nova classificação climática do Estado do Rio Grande do Sul. Revista Brasileira de Agrometeorologia, Piracicaba, v. 8, n. 1, p. 141-150, 2000.
MANEYRO, R.; NAYA, D. E.; ROSA, I.; CANAVERO, A.; CAMARGO, A. Diet of the South American frog Leptodactylus ocellatus (Anura: Leptodactylidae) in Uruguay. Iheringia, Série Zoologia, Porto Alegre, v. 94, n. 1, p. 57-61, 2004.

PIANKA, E. R. The structure of lizard communities. Annual Review of Ecology and Systematics, Palo Alto, v. 4, p. 53-74, 1973.

PINKAS L.; OLIPHANT, M.; IVERSON, Z. Food habitat of albacore bluefin, tuna and bonito in California waters. California Department Of Fish and Game's Fish Bulletin, California, v. 152, p. 1-350, 1971.

RODRIGUES, E. A. S.; DIAS, I. R.; CAVALCANTE, K. P.; BRANCO, S. M. J.; SILVA JÚNIOR, E. M. S.; SOLÉ, M. Análise da dieta de Leptodactylus spixi (Anura: Leptodactylidae) de uma cabruca do sul da Bahia. In: CONGRESSO DE ECOLOGIA DO BRASIL, VIII, 2007, Caxambu. Anais... Caxambu: SEB, 2007. Versão eletrônica.

SANABRIA, E. A; QUIROGA, L. B; ACOSTA, J. C. Dieta de Leptodactylus ocellatus (Linnaeus, 1758) (Anura: Leptodactylidae) en un humedal del oeste de Argentina. Revista Peruana de Biología, v. 12, n. 3, p. 472-477, 2005.

SBH - Sociedade Brasileira de Herpetologia. Brazilian amphibians - List of species. Disponível em: $<$ http://www.sbherpetologia.org. br>. Acesso em: 15 jan. 2011.

SOLÉ, M.; DIAS, I. R.; RODRIGUES, E. A. S.; MARCIANO-JR, E.; BRANCO, S. M. J.; CAVALCANTE, K. P.; RÖDDER, D. Diet of Leptodactylus ocellatus (Anura: Leptodactylidae) from a cacao plantation in southern Bahia, Brazil. Herpetology Notes, Pisa, v. 2, p. 9-15, 2009.

STRÜSSMANN, C.; VALE, M. B. R.; MENEGHINI, M. H.; MAGNUSSON, W. E. Diet and foraging mode of Bufo marinus and Leptodactylus ocellatus. Journal of Herpetology, Salt Lake City, v. 18, n. 2, p. 138-146, 1984.

TEIXEIRA, R. L.; VRCIBRADIC, D. Diet of Leptodactylus ocellatus (Anura, Leptodactylidae) from coastal lagoons of southeastern Brazil. Cuadernos de Herpetologia, Tucumán, v.17, n. 1-2, p. 111-118, 2003.

TOFT, C. A. Feeding ecology of Panamanian litter anurans: patterns in diet and foraging mode. Journal of Herpetology, Salt Lake City, v. 15, n. 2, p. 139-144, 1981. 